\title{
A feasibility cluster randomised controlled trial of a preschool obesity prevention intervention: ToyBox-Scotland
}

Stephen Malden ${ }^{1 *}$, John. J. Reilly ${ }^{1}$, Ann-Marie Gibson ${ }^{1}$, Farid Bardid ${ }^{2,3}$, Carolyn Summerbell ${ }^{4}$, Marieke De Craemer ${ }^{3}$, Greet Cardon ${ }^{3}$, Odysseas Androutsos ${ }^{5}$, Yannis Manios ${ }^{5}$ and Adrienne Hughes ${ }^{1}$

\begin{abstract}
Background: High levels of childhood obesity have been observed globally over the last three decades. Preschools are promising settings to implement obesity prevention interventions in the early years. The aim of this study was to test the feasibility of a cluster randomised controlled trial of the ToyBox-Scotland preschool obesity prevention intervention.

Methods: Six preschools in predominantly deprived areas of Glasgow, UK, were randomised to either the ToyBox intervention ( $n=3)$ or usual curriculum control group $(n=3)$. The intervention ran for 18 weeks from March-June 2018, and consisted of practitioner-led physical activity and sedentary behaviour sessions in preschools, with an additional interactive home component. Primary outcome measures were intervention fidelity, recruitment rates, attrition rates, and compliance with trial procedures. Secondary outcomes were body mass index (BMI) $z$-score, bioelectrical impedance analysis (BIA), objectively measured physical activity and sedentary time via activPAL accelerometer, and parent-reported home eating, snacking, and water consumption.
\end{abstract}

Results: The preschool component of the intervention was implemented with high fidelity (64\%), while the home component was implemented with low fidelity (41\%). A cluster-level recruitment rate of $10 \%$ was achieved, and the individual-level recruitment rate was 18\% (42/233 children, mean age 4.4 years; 17 girls). The attrition rate was 14\%, and compliance rates varied considerably by the outcome. Compliance was highest for BMI (86\%), while 19\% of the sample returned valid accelerometer data for both baseline and follow-up and the parental questionnaire response rate was 23\%. Both intervention and control groups showed small increases in BMI $z$-scores at follow-up of 0.02 and 0.06 , respectively. Both groups had small decreases in physical activity and increases in sedentary time at follow-up.

Conclusions: Before progression to an effectiveness trial, additional procedures should be considered to improve recruitment rates, compliance with outcome measures, and implementation of the home-based component of the ToyBox-Scotland intervention.

Trial registration: ISRCTN12831555

Keywords: Childhood obesity, Feasibility, Physical activity, Sedentary behaviour, Prevention

\footnotetext{
* Correspondence: stephen.malden@strath.ac.uk

'Physical Activity for Health Group, School of Psychological Sciences and Health, University of Strathclyde, Graham Hills Building, 40 George Street, Glasgow G1 1XP, UK

Full list of author information is available at the end of the article
}

(C) The Author(s). 2019 Open Access This article is distributed under the terms of the Creative Commons Attribution 4.0 International License (http://creativecommons.org/licenses/by/4.0/), which permits unrestricted use, distribution, and reproduction in any medium, provided you give appropriate credit to the original author(s) and the source, provide a link to the Creative Commons license, and indicate if changes were made. The Creative Commons Public Domain Dedication waiver (http://creativecommons.org/publicdomain/zero/1.0/) applies to the data made available in this article, unless otherwise stated. 


\section{Background}

High levels of childhood obesity are evident globally [1], with obesity in the early years being linked to elevated total and low-density lipoprotein (LDL) cholesterol in children as young as 3 years [2]. While the causes of childhood obesity are multifactorial, research has demonstrated causal links between excess weight and energy balance-related behaviours such as physical activity (PA), sedentary behaviour (SB), and diets high in fat and refined sugars $[3,4]$.

Preschools offer a potentially effective setting to address obesity prevention, and a number of interventions have targeted such settings with varying levels of success [5-7]. Specifically, multicomponent interventions which target $\mathrm{PA}, \mathrm{SB}$, and diet both in the preschool and home environment tend to show the most promise with regards to improving energy-balance-related behaviours and preventing obesity in young children $[8,9]$.

One such intervention is called ToyBox, which employs teacher-led sessions to target energy-balance related behaviours at preschool, while behaviours in the home environment are targeted using informative materials for parents [10]. The intervention, when tested in six countries across Europe, led to significant improvements in water consumption [11], PA, SB [6], and family-related determinants of unhealthy snacking [12]. The intervention has subsequently been adapted for use in other European countries, Malaysia [13], and most recently in Scotland [14], where context-specific adaptations were made to the intervention content and delivery to suit the social and cultural needs of Scottish preschools. However, prior to testing an intervention in an effectiveness trial, the UK Medical Research Council recommends that a feasibility study should be conducted first as it is considered an integral aspect of intervention development and evaluation [15]. Therefore, the aim of this study was to test the feasibility of a cluster randomised controlled trial (RCT) of the ToyBox-Scotland preschool obesity prevention programme to inform the design of a future full-scale RCT.

\section{Methods}

\section{Study design}

This study was designed in accordance with the CONSORT statement's extension to randomised pilot and feasibility trials [16]. This study had a cluster RCT design consisting of an intervention group (three preschools) and a control group (three preschools). As this was a feasibility study, no sample size calculation was undertaken. As all participating preschools were similar in size and demographics, no matching was undertaken prior to randomisation. An independent researcher was presented with six identical envelopes by a member of the research team not involved in data collection or analysis. Each envelope contained the name of the participating preschools. They were then instructed to select three envelopes at random to be control preschools. The remaining envelopes were assigned to the intervention group. Data were collected between January and June 2018. This study was approved by the University of Strathclyde's School of Psychological Sciences and Health Ethics Committee.

\section{Setting, sampling, and participants}

Glasgow is the largest urban area in Scotland and is one of the most socioeconomically deprived areas in Western Europe, with over a third of the cities' children estimated to be living in poverty [17]. A Glasgow City Council representative contacted a convenience sample of all local authority preschools in the Glasgow City area via email to seek expressions of interest to participate $(n=112)$. Eleven preschools expressed an interest to participate in the study, of which 6 were selected based on similarities in demographics, size, and socio-economic status (SES). Head teachers at participating preschools were visited by the study manager and provided with information sheets and consent forms, which they distributed to parents/caregivers of all 3-5-year-old children at their preschools. Children were excluded from the study if they had a health condition that would significantly limit their ability to participate in the intervention or if parental consent was not provided. The intervention was delivered to all 3-5-year-old children in the intervention preschools. All six preschools received $£ 200$ after the completion of the study to offset any participation costs.

\section{Intervention}

Prior to the commencement of the present study, the original ToyBox intervention [10] was adapted to suit the Scottish preschool setting. The process of adaptation is described in detail elsewhere [14]. Briefly, alterations to the number of PA and SB sessions were made to reflect the focus on child-led learning in Scottish preschool practice and classroom manuals were re-written to reflect the language used in the Scottish education system. Additional adaptations included the removal of the preschool-based eating/snacking and water consumption components and the addition of more interactive parent/child activities to address energy-balance behaviours (i.e. eating/snacking, water consumption, PA, and $\mathrm{SB}$ ) in the home environment. All adaptations were undertaken using a co-creation approach [18], whereby relevant stakeholders and an experienced early years' practitioner assisted the research team with the adaptation process.

Preschools receiving the ToyBox-Scotland intervention were provided with $\mathrm{a} \sim 2.5 \mathrm{~h}$ practitioner training session prior to the intervention. Preschools received a box with 
additional classroom materials such as kangaroo hand puppets and classroom activity guides. Classroom activity guides offered detailed instructions on the delivery of $\mathrm{PA}$ and SB sessions, and the setup of the classroom environment to encourage PA and active play and to reduce SB. Practitioners were given autonomy to deliver the intervention throughout the day when time allowed, but were encouraged to deliver activities for a total of $1 \mathrm{~h}$ per week, and gradually increase this as the intervention progressed. Parents received a sticker wallchart and bi-weekly activity packs containing tip cards, newsletters, interactive games and sticker incentives to award to their child after they completed each of the home-based activities. The intervention was delivered for 18 weeks, where $\mathrm{PA}$ and SB were targeted in both the preschool and home environment, and eating and water consumption was targeted in the home environment, as detailed in Table 1.

\section{Procedures and outcomes}

Participants were measured at two time-points by one researcher (SM) and a fieldwork assistant, who were both trained in the measures. Baseline assessment was undertaken in late January/early February 2018, with follow-up measurement taking place 15-17 weeks later. An early years' practitioner at each preschool was present for all data collection procedures to prepare and accompany children through data collection and assist with any issues. Although parental consent was collected for all participating children, child assent was obtained from each child on the measurement day, and children who did not want to participate in any of the data collection procedures were not obliged to do so. The primary outcome measure for this study was the feasibility of the intervention and trial. Therefore, the primary outcomes of interest were recruitment rates, attrition rates, implementation fidelity and compliance rates with data collection procedures. A number of secondary outcomes were also assessed, detailed below.

\section{Implementation fidelity}

Implementation fidelity refers to the extent to which an intervention is implemented as intended by those who developed it [19]. Fidelity was assessed in both the preschool and home environments using the following methods:

\section{Preschool component}

In order to assess implementation fidelity at preschools, practitioners were supplied with a monthly logbook for the duration of the programme, which was adapted from the original ToyBox study logbook [20]. For each month that the intervention was delivered $(n=4)$, practitioners completed five-point Likert scales, which assessed the extent to which the components of the intervention were delivered. Namely, changes to the classroom environment, children performing health behaviours, and classroom experiences.

\section{Home component}

Practitioners recorded how many eligible children were supplied with home activity packs each month, while parents/caregivers received a post-intervention questionnaire (Additional file 1). This questionnaire comprised yes/no questions and 5-point Likert scales, with questions designed to identify to what extent the parents/ caregivers received and engaged with the intervention materials at home.

\section{Secondary outcome measures Body mass index}

Height and weight were measured by the same researcher with children wearing light clothing and with shoes removed. Height was measured using a stadiometer (Marsden, UK) to the nearest $0.1 \mathrm{~cm}$, and weight was measured using an electronic scale (Tanita, Amsterdam, Netherlands) to the nearest $0.1 \mathrm{~kg}$. Measurements were conducted in a private meeting room, with children measured in small groups of 3-4 at a time. Only the researcher was able to see the readings. Both height and weight measurements were taken twice and the average calculated. Body mass index (BMI) $z$-scores were calculated from the height and weight data using standardised methods [21]. Children aged $\geq 4$ years were categorised using UK90 growth reference charts [22], while the WHO growth reference was used to calculate $z$-scores for 3-year-olds [23]. Children $<85$ th percentile were classified as normal weight, $\geq 85$ th percentile as overweight, and $\geq 95$ th percentile as obese.

\section{Objectively measured PA}

PA was measured objectively using the activPAL accelerometer (model ActivPAL3; PAL Technologies Ltd.,

Table 1 Intervention structure for ToyBox-Scotland

\begin{tabular}{|c|c|c|c|c|c|c|c|c|c|c|c|c|c|c|c|c|}
\hline & \multicolumn{7}{|c|}{ First focus } & \multicolumn{9}{|c|}{ Repetition } \\
\hline & \multicolumn{2}{|l|}{3 weeks } & \multicolumn{2}{|c|}{3 weeks } & \multicolumn{2}{|c|}{3 weeks } & 3 weeks & & \multicolumn{2}{|l|}{2 weeks } & \multicolumn{2}{|c|}{2 weeks } & \multicolumn{2}{|l|}{1 week } & \multicolumn{2}{|c|}{1 week } \\
\hline Preschool & PA & & $S B$ & & PA & & SB & & PA & & SB & & PA & & SB & \\
\hline Home & WC & PA & ES & SB & WC & PA & ES & SB & WC & PA & ES & SB & WC & PA & ES & SB \\
\hline
\end{tabular}

ES eating and snacking, $P A$ physical activity, $S B$ sedentary behaviour, $W C$ water consumption 
Glasgow, UK). The activPAL is a small wearable device that is attached to the front of the mid-thigh and measures postural information, which can be categorised into sitting/lying, standing and moving/stepping activity [24]. Once attached, the device can be worn continuously for 7-10 days. Participants were fitted with the activPAL by assisting early-years practitioners under the instruction of the researchers. Parents were instructed to leave the activPAL on for seven consecutive days, with 3 days wear time [25] considered valid for this study.

\section{Body composition}

Supine arm-to-leg bioelectrical impedance analysis (BIA) was used to measure fat mass and fat-free mass with the Bodystat 1500 (Bodystat Ltd., Douglas, Isle of Man). Measures were taken twice and the average was calculated. A full description of the procedures and formulae to use with this age group is available elsewhere [26].

\section{Objectively measured $S B$}

The activPAL was used to assess sedentary time during waking hours using the same procedure as PA described above [27]. Periods of nighttime sleep were differentiated from waking sedentary time by studying the raw data files to determine when no significant changes in axis of movement (from sitting/lying to standing) were detected from one 24-h period to the next, as such observations indicate the participant is asleep during these times.

\section{Home eating, snacking, water consumption, and screen time}

The Primary Caregiver Questionnaire (PCQ) and the Food Frequency Questionnaire (FFQ) used in the original ToyBox study were adapted for use in the present study. Specifically, the number of questions in each were reduced as recommended by stakeholders during development meetings (questions related to maternal/postnatal nutrition were removed from the FFQ and family history questions removed from the PCQ). The questionnaires required parents to provide information on their children's fruit/vegetables, confectionary, water, and sugar-sweetened beverage consumption in addition to the use of screen devices and sleep patterns. Questionnaires were supplied to preschools by the research team in paper format and were then distributed to participating parents when they collected their children by preschool staff. Full details regarding the development, validity, and test-retest reliability of the questionnaires are reported elsewhere [28-30].

\section{Analysis}

In order to assess fidelity of implementation in this study, scoring systems used by Verloigne et al. [31] and Pinket et al. [32] were adapted and used to assign codes to participant's logbook and questionnaire responses that indicated the level of implementation. For dichotomous items, a positive response (yes) was coded as 1 , while a negative response (no) received a 0 . For Likert scale items, a response of either 4 (agree/often) or 5 (strongly agree/always) was coded as 1 , while all other responses (1-3, strongly disagree/never; disagree/not often; neither agree nor disagree/sometimes) were coded as 0 . Total implementation fidelity scores of 72 and 11 were available for practitioners and parents, respectively. Accelerometer data were entered into PAL analysis software and mean daily time spent in PA, step count, sedentary time, and sleep were computed for all devices which met the 3-day valid wear-time cut-off. To calculate and categorise participant's weight status from the height and weight measurements, data was entered into the LMS Growth add-in for Microsoft Excel to generate $z$-scores and percentile scores. As this is a feasibility study, the use of inferential statistics and effectiveness testing is not recommended due to the small sample size and the preliminary nature of the outcomes measured [33]. Instead, descriptive statistics were used to assess feasibility parameters such as fidelity of implementation, recruitment, retention and attrition rates from baseline to follow-up, presented as proportions. High, medium, and low fidelity was classified as an overall implementation score of $\geq 60 \%, \geq 50<60 \%$, and $<50 \%$ respectively, following recommendations from similar studies [19]. For the secondary outcomes, means \pm standard deviations are presented, with the mean change from baseline to follow-up for each outcome calculated along with 95\% confidence intervals where appropriate. Process evaluation data (e.g. teacher logbooks and parental feedback surveys) were analysed prior to outcome data, as recommended by current guidelines on process evaluation [34].

\section{Results}

\section{Feasibility of trial recruitment and retention}

Eleven out of 112 preschools responded positively to an invitation to take part in the study (cluster-level response rate $=10 \%$ ). A total of 233 consent forms were distributed, of which 42 children (mean age $4.4 \pm 0.46$ years; 17 girls) provided parental consent and completed baseline assessment (individual-level recruitment rate = $18 \%$ ) before preschools were randomised to the ToyBoxScotland intervention arm ( 3 centres; $n=26 ; 10$ girls) or the usual curriculum control arm ( 3 centres; $n=16 ; 7$ girls). See Fig. 1 for CONSORT flow diagram.

\section{Intervention fidelity}

All intervention preschools returned complete logbooks for the 4 month study period. Overall, the intervention was implemented with high fidelity across the three intervention preschools (64\%), with one preschool 


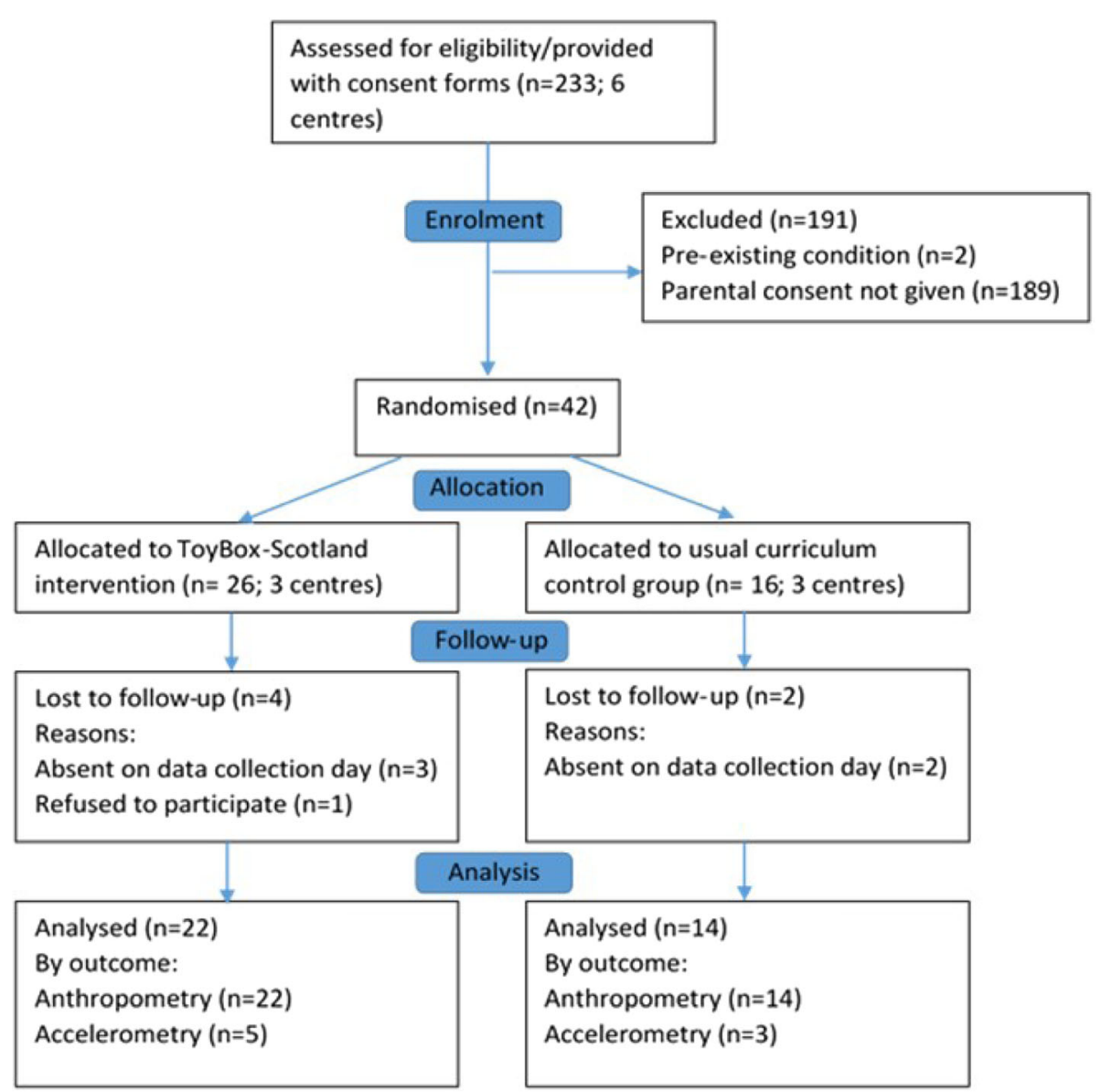

Fig. 1 CONSORT flow diagram detailing trial recruitment and retention for ToyBox-Scotland

implementing with medium fidelity (52\%), and two with high fidelity (79\% and 61\%). Intervention components relating to PA were generally implemented with higher fidelity than SB components (Table 2). Twenty-six parents returned post-intervention feedback surveys, of which seven were incomplete and excluded (19/125; 15\% response rate). The overall implementation score for the home component of the intervention was low (41\%) based on post-intervention survey responses. Specific preschool implementation scores from practitioners' logbook data are detailed in Table 2 .

\section{Participation in outcome measures \\ Anthropometry}

Eighty-six percent (36/42) of participants provided valid height and weight measurements at baseline and followup. Five children were absent on the follow-up data collection day, and one did not want to participate.

\section{Body composition}

Six children out of 42 (14\%) adequately complied with the BIA protocol at baseline. However, the readings from these were not valid as children did not adhere to the protocol and the use of BIA was not carried forward to follow-up.

\section{Accelerometry}

Fifty-two percent of the participants provided valid accelerometer data at baseline $(n=22)$. Reasons for invalid measurement were as follows: device malfunction $(n=$ $8)$; removed before valid wear-time due to skin irritation $(n=7)$; device loss $(n=5)$. Only participants who supplied valid data at baseline were fitted with an accelerometer at follow-up. Nineteen percent of the original sample returned valid accelerometer data at follow-up. Reasons for loss of data at follow-up were removed before valid wear time cut-off (no specific reason provided; $n=5)$; device lost $(n=4)$; child absent on data collection day $(n=5)$.

\section{Parental questionnaires (demographics/family history, dietary habits, screen time)}

Early years practitioners distributed the PCQ and FFQ to the parents/caregivers of all participating children at 
Table $\mathbf{2}$ Implementation fidelity score logbook items and responses

\begin{tabular}{|c|c|c|c|c|c|}
\hline \multirow[t]{2}{*}{ Component } & \multirow[t]{2}{*}{ Logbook question } & \multicolumn{4}{|c|}{$\begin{array}{l}\text { Scoring and results (\% coded as } 1 \\
\text { over the } 4 \text { months) }\end{array}$} \\
\hline & & $\begin{array}{l}\text { PS A } \\
(\%)\end{array}$ & $\begin{array}{l}\text { PS B } \\
(\%)\end{array}$ & $\begin{array}{l}\text { PS C } \\
(\%)\end{array}$ & $\begin{array}{l}\text { Overall (\%) } \\
\text { (fidelity score) }\end{array}$ \\
\hline \multirow[t]{4}{*}{ Preschool environment } & $\begin{array}{l}\text { Were the number of chairs in the classroom reduced to encourage standing } \\
\text { play?* }\end{array}$ & 0 & 0 & 0 & 0 (low) \\
\hline & $\begin{array}{l}\text { Was equipment and space appropriately arranged for physical activity sessions } \\
\text { every day of the week?* }\end{array}$ & 100 & 50 & 100 & 83 (high) \\
\hline & $\begin{array}{l}\text { Was the classroom appropriately arranged for movement breaks every day of } \\
\text { the week?* }\end{array}$ & 100 & 75 & 0 & 58 (med) \\
\hline & Were any movement corners set up and made available to the children?* & 75 & 0 & 0 & 25 (low) \\
\hline \multirow{4}{*}{$\begin{array}{l}\text { Children performing the health } \\
\text { behaviours }\end{array}$} & Did you regularly remind children to drink water?* & 100 & 100 & 100 & 100 (high) \\
\hline & Did you remind children to drink water after they have been active?* & 100 & 100 & 100 & 100 (high) \\
\hline & $\begin{array}{l}\text { Did you remind children to bring healthy snacks from home (or remind the } \\
\text { catering service/canteen to provide healthy snacks to children?)* }\end{array}$ & 100 & 100 & 100 & 100 (high) \\
\hline & $\begin{array}{l}\text { How much time did you devote to physical activity sessions on an average } \\
\text { weekly basis this month? }\end{array}$ & 100 & 75 & 100 & 92 (high) \\
\hline \multirow[t]{7}{*}{ Classroom experiences } & 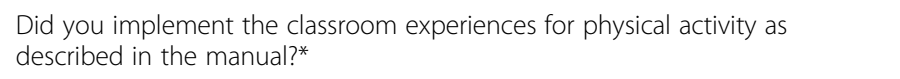 & 100 & 50 & 100 & 83 (high) \\
\hline & $\begin{array}{l}\text { Did you devote on average at least } 1 \mathrm{~h} \text { per week to the classroom activities } \\
\text { for physical activity as described in the manual?*}\end{array}$ & 100 & 50 & 100 & 92 (high) \\
\hline & $\begin{array}{l}\text { Did you devote on average at least } 1 \mathrm{~h} \text { per week to the classroom activities } \\
\text { for sedentary behaviour as described in the manual?** }\end{array}$ & 50 & 0 & 0 & 17 (low) \\
\hline & $\begin{array}{l}\text { Which classroom activity(ies) regarding physical activity did you implement } \\
\text { this month? }{ }^{+}\end{array}$ & 100 & 50 & 50 & 67 (high) \\
\hline & $\begin{array}{l}\text { How many of the little kangaroo stories for physical activity did you use this } \\
\text { month? }{ }^{+}\end{array}$ & 19 & 0 & 25 & 11 (low) \\
\hline & $\begin{array}{l}\text { How many of the little kangaroo stories for sedentary behaviour did you use } \\
\text { this month? }{ }^{+}\end{array}$ & 8 & 0 & 0 & 3 (low) \\
\hline & $\begin{array}{l}\text { Which classroom activity(ies) regarding sedentary behaviour did you } \\
\text { implement this month? }^{+}\end{array}$ & 100 & 25 & 0 & 42 (low) \\
\hline \multirow[t]{4}{*}{$\begin{array}{l}\text { Delivery of home materials and } \\
\text { engagement with parents }\end{array}$} & $\begin{array}{l}\text { Did you provide parents with the pre-prepared home activity packs when } \\
\text { these were delivered to the nursery?*}\end{array}$ & 100 & 75 & 100 & 92 (high) \\
\hline & $\begin{array}{l}\text { Estimate the number of parents to whom you directly delivered programme } \\
\text { materials. If you did }{ }^{+} \text {(total } 125 \text { children) }\end{array}$ & 100 & 85 & $100 \%$ & 95 (high) \\
\hline & $\begin{array}{l}\text { Estimate the number of parents for whom you spent time to explain the } \\
\text { purpose of the material and encourage them to follow the recommendations } \\
\text { of the material }{ }^{+} \text {(total } 125 \text { children) }\end{array}$ & 11 & 7 & 15 & 12 (low) \\
\hline & Total aggregate scores (\% responses coded as 1. Total available points $=72$ & 79 & 52 & 61 & $\begin{array}{l}\text { Overall } \\
\text { score }=64\end{array}$ \\
\hline
\end{tabular}

This form was repeated four times, once for each month the intervention was delivered

*Scoring determined by 5 -point scale, " $1=$ never, 2 = not often, $3=$ sometimes, $4=$ often, $5=$ always" $\geq 4=1 ; \leq 3=0$

${ }^{+}$Scoring determined by a "yes $/ \mathrm{no}^{\prime}$ response or a numerical response. $Y e s=1 ; n o=0$. Numerical responses equate to $\geq 60 \%=1 ;<60 \%=0$. $P S=$ preschool

baseline $(n=42)$. Twenty-three percent of parents returned completed questionnaires for both baseline and follow-up $(n=10)$.

\section{Behavioural and health outcomes}

For the 22 participants that provided valid accelerometer data at baseline, mean daily minutes spent in PA was 163 (30) and 151 (40) for the intervention and control groups respectively (mean daily steps of 11,437 (2351) for the intervention group, and 10,827 (2895) for the control group). The intervention group spent an average of 420 (72) $\mathrm{min} /$ day sedentary, and the control group spent 396 (72) min sedentary. Table 3 summarises the results for participants that completed measurement at baseline and follow-up. Small increases in BMI $z$-score were observed for both groups; however, the increase was larger in the control group. Both groups showed reductions in mean daily minutes spent in PA and daily steps from baseline to follow-up, with the larger decreases observed in the intervention group. Sedentary time per day increased by almost $30 \mathrm{~min}$ and $10 \mathrm{~min}$ in the intervention and control groups, respectively. 
Table 3 Behavioural and health outcomes at baseline and follow-up

\begin{tabular}{|c|c|c|c|c|c|c|}
\hline \multirow[t]{2}{*}{ Pre and post- results } & \multicolumn{2}{|l|}{ Intervention } & \multirow[b]{3}{*}{ Mean change $(95 \% \mathrm{Cl})$} & \multicolumn{2}{|l|}{ Control } & \multirow[b]{3}{*}{ Mean change $(95 \% \mathrm{Cl}$ ) } \\
\hline & Baseline & Follow-up & & Baseline & Follow-up & \\
\hline Measurement & Mean (SD) & Mean (SD) & & Mean (SD) & Mean (SD) & \\
\hline BMI z-score & $0.41(1.16)$ & $0.43(1.09)$ & $0.02(-0.11,0.15)$ & $0.35(1.17)$ & $0.41(1.07)$ & $0.06(-0.04,1.05)$ \\
\hline Total daily PA (min) & $165(58)$ & $151(27)$ & $-14(-87,115)$ & $144(41)$ & $143(42.1)$ & $-1(-117,121)$ \\
\hline Total daily ST (min) & $428(62)$ & $456(100)$ & $28(-174,120)$ & $407(81)$ & $417(52)$ & $10(-216,192)$ \\
\hline Total daily steps (count) & $12,035(4084)$ & $10,718(2020)$ & $-1316(-5818,8451)$ & $10,221(3004)$ & $10,017(3240)$ & $-204(-8235,8644)$ \\
\hline
\end{tabular}

\section{Discussion}

This study investigated the feasibility of a cluster randomised controlled trial of the adapted ToyBox-Scotland childhood obesity prevention intervention. Participating preschools were willing to be randomised, and trial procedures and pre-school-based intervention components were deemed feasible by preschool staff. The intervention was implemented with high fidelity within the preschool. However, implementation of the home component was lower, a finding that is commonly reported in other school-based interventions with home components [32, 35]. The cluster-level recruitment rate of $10 \%$ in this study is lower than that achieved in similar feasibility studies targeting young children [36-38], as was the observed individual-level recruitment rate of $18 \%$ [36, 37, 39, 40]. Conversely, the overall trial retention rate of $86 \%$ ( $14 \%$ attrition rate) is similar to or higher than other trials $[6,36]$. However, within those participants that were retained from baseline to followup, the collection of valid measures varied considerably by the outcome.

At $41 \%$, the level of implementation observed in the home environment was low. Additionally, the low postintervention survey response rate of $15 \%$ indicates that implementation was even lower, as it is unlikely that non-respondents engaged highly with the intervention. These findings are unsurprising, considering the home environment has previously been identified as one of the more challenging settings to implement obesity prevention interventions in [41], particularly in low SES groups. While overall preschool intervention fidelity was high, it was apparent from logbook responses that PA components of the intervention were implemented at a higher level than SB components (Table 2). This finding was also observed in the original ToyBox study, where SB implementation scores were relatively low across multiple intervention sites within the six participating regions [35]. Considering these findings, we adapted the programme accordingly, reducing the number of more time-consuming activities in the intervention [14]. Despite this, the relatively poor implementation scores observed for SB activities highlights that further adaptation may be needed for the SB component and for the homebased components as a whole.
In the Belgian ToyBox study cohort, recruitment involved a personal visit by a member of research staff to all eligible preschools $(n=97)$, which resulted in a cluster-level recruitment rate of $28 \%$ [6]. Additionally, the study achieved an individual-level recruitment rate of $39 \%$, utilising the same procedures of staffadministered information sheets and consent forms to parents as our study. However, it is important to consider the differences in demographics recruited between the two studies. The Belgian study recruited participants from 27 preschools, for which $55 \%$ were classed as either medium or high SES. In our study, all but 1 of the 6 recruited preschools were within the $20 \%$ most deprived areas in Scotland. An abundance of research has demonstrated that more deprived population groups are more difficult to recruit into trials and are also more likely to drop out than participants in higher SES groups [42-44]. Therefore, the lack of medium-high SES preschools recruited to this present trial may have negatively impacted on the recruitment rates achieved. Considering these observations, in any future trial, it may be of benefit to conduct personal visits to eligible preschools to improve the school recruitment rates, and also target preschools in areas of high, medium, and low SES, using different strategies to recruit participants from deprived populations to account for the lower recruitment rates observed within these areas.

Eighty-six percent of the original sample completed height and weight measures in this study, which is comparable to anthropometric measurement rates of similar studies $[7,39,45,46]$, indicating that these procedures are feasible with this population group. However, we encountered significant issues with the collection of valid BIA data at baseline, and measurement of this outcome was not carried forward to the follow-up. Obtaining accurate BIA readings requires participants to follow a strict protocol consisting of a period of fasting and restricted PA prior to and during the collection of the readings, which was not possible with this sample. Furthermore, there are conflicting arguments in the literature regarding the validity of such methods with children [47]. Our intended use of BIA was to further validate BMI $z$-scores with another measure, as BMI is a crude proxy measure for adiposity $[48,49]$. Therefore, in 
any future trial, it may be beneficial to use other anthropometric measures alongside BMI such as waist circumference, skinfold thickness or hip-waist ratio which have proved feasible in other trials [6, 45], and show high agreement with BMI estimates.

With regard to accelerometry, a number of factors prevented the collection of valid wear-time data at both baseline and follow-up. Studies that use objective measures of free-living PA and SB in children offer conflicting findings with regards to compliance with measurement procedures. A recent review of attrition rates and noncompliance with accelerometers in childhood PA trials found that non-compliance at follow-up ranged from 3 to 70\% across 23 studies [50]. Conversely, Jones et al. (2011) used Actigraph accelerometers worn for two consecutive days in a pilot RCT of a fundamental movement skills and PA intervention in preschool children, reporting high adherence rates of $96 \%$ and $97 \%$ for baseline and 6-month follow-up, respectively [51], indicating that reduced weartime may increase compliance. However, a similar study used the Actigraph for 7 days in pre-schoolers and achieved an $86 \%$ adherence rate, indicating additional factors likely influence accelerometer compliance [51].

While some unavoidable reasons for loss of data such as device malfunction/loss and child absence in our study reflect issues commonly encountered in accelerometer studies mentioned previously, a number of reasons for the early removal of the device are specific to the activPAL accelerometer that we used. Specifically, a small number of parents reported that their child developed a rash due to wearing the waterproof medical adhesive which attached the device to the leg. While this was likely a harmless sweat rash, a recent study also found that adolescents who were asked to record their reasons for removing the activPAL in a compliance study cited skin irritation as the primary reason for early removal [53], a finding which is supported by another study on pre-schoolers [24]. These issues could be addressed by reducing the required wear time and improving communication with parents so that they are aware of how to reattach the device or how to distinguish a sweat rash from an allergic reaction. Alternatively, the activPAL showed to be comparable with other wearable devices that are perhaps less invasive and more participant-friendly in their attachment methods [24, 27] which may be valid alternatives in further trials. Regardless of the device used, creating better links with parents from recruitment through to follow-up will likely facilitate the collection of a higher proportion of valid accelerometer data and should be a priority in any future trial. A number of trials have reported favourable results from the use of reminder texts/phone calls to parents or the provision of small monetary incentives for the safe return of accelerometers [39].
Parental response rates to the FFQ and PCQ questionnaires were also considerably lower than rates observed in the original ToyBox study and in other trials [54]. Although efforts to reduce the length of these questionnaires were taken prior to the trial commencing, additional adaptation may be needed to increase the response rates. Due to the need to calculate portion sizes and recall dietary patterns, the FFQ can be time-consuming to complete [55, 56], and parents may not know what their child has eaten while at preschool. This, coupled with the relatively low levels of adult literacy observed in the areas which we sampled [57], may have negatively impacted our questionnaire response rates. Therefore, other more time-efficient alternatives to the FFQ should be explored [56].

This study had a number of limitations. Firstly, due to the low preschool-level response rate, it was only possible to sample preschools located in areas of lower SES, which limits the generalisability of our findings to the wider Scottish preschool population. However, this issue is somewhat unavoidable when conducting research within Glasgow, which has a significantly higher concentration of deprived localities than the rest of Scotland's local authority areas [58]. This issue could be addressed in the future through the use of stratified sampling, which would allow for the assessment of differences in intervention effectiveness between SES groups. Despite this, due to the marked social patterning observed in obesity risk, there is a need to target interventions at low SES groups [59]; therefore, the lessons learned from this study will be of value during the design of future trial procedures.

Secondly, while the aim of this study was to test feasibility, and not to test effectiveness, the low response rates to questionnaires and non-compliance with accelerometer measures makes it difficult to determine any direction of intervention effect, which would have indicated whether the intensity of the intervention was likely to be sufficient. Despite these limitations, the data gathered during this trial are sufficient to assess the feasibility of the study design and the fidelity of the intervention, which will assist with the development of effectiveness and efficacy trials. Another important aspect of feasibility testing not addressed in this present paper is the acceptability of the intervention and trial procedures. Items pertaining to acceptability were included in both teacher and parent post-intervention questionnaires, and qualitative interviews and focus groups were conducted with both parents and practitioners, respectively. A separate paper will present these data and explore acceptability by identifying important barriers and facilitators to implementation. 


\section{Conclusions}

The findings of this study indicate that although aspects of this cluster RCT of the ToyBox Scotland intervention were feasible, more efforts to increase recruitment rates, accelerometer compliance, and questionnaire response rates should be further investigated before progression to any future trial. Specifically, more development activities should be undertaken with preschools, parents, and children to ensure that both the intervention components and the methods of evaluation are appropriate and acceptable before progressing to further effectiveness testing.

Testing feasibility before progressing to a fully powered trial is an effective way to identify issues with sampling/recruitment, implementation fidelity, trial design, and methods of outcome measurement. This study, coupled with the results of an ongoing investigation of the barriers and facilitators to implementation of the intervention, will further highlight priorities for further adaptation prior to any future trial of the ToyBox Scotland intervention.

\section{Supplementary information}

Supplementary information accompanies this paper at https://doi.org/10. 1186/s40814-019-0521-7

Additional file 1. The ToyBox preschool health programme parent/ guardian feedback form.

\section{Abbreviations}

BIA: Bioelectrical impedance analysis; BMI: Body mass index; FFQ: Food frequency questionnaire; LDL: Low-density lipoprotein; PA: Physical activity; PCQ: Primary caregiver questionnaire; SB: Sedentary behaviour; SES: Socioeconomic status; ST: Sedentary time; WHO: World Health Organisation

\section{Acknowledgements}

The authors thank all preschool staff, parents, and children who participated in the study. The authors thank Kay Hamilton and Julie Martin from Glasgow City Council Education services for their assistance with recruitment to the study.

\section{Authors' contributions}

SM managed the study and wrote the first draft of the manuscript. JJR, AH, and $A M G$ assisted with intervention adaptation, ethics applications, and study design and writing of the second draft of the manuscript. FB, MDC, CS, GC, OA, and YM substantially contributed to the final draft of the manuscript. All authors read and approved the final manuscript.

\section{Funding}

Research relating to this study was funded by the Cunningham Trust. The funders had no role in study design, data collection, data analysis, interpretation of findings, or preparation of this manuscript.

\section{Availability of data and materials}

As data were collected from local authority preschool children, public availability of the datasets is restricted by Glasgow City Council data protection policies. However, datasets relating to this study are available from the corresponding author upon reasonable request.

\section{Ethics approval and consent to participate}

This study was granted approval by the University of Strathclyde's School of Psychological Sciences and Health ethics committee. All parents/caregivers of participating children provided written informed consent for their child to participate in the study.

\section{Consent for publication}

All participants in this study gave their consent for results to be published as part of the informed consent process. No individual data are presented in this paper.

\section{Competing interests}

The authors declare that they have no competing interests.

\section{Author details}

${ }^{1}$ Physical Activity for Health Group, School of Psychological Sciences and Health, University of Strathclyde, Graham Hills Building, 40 George Street, Glasgow G1 1XP, UK. ${ }^{2}$ School of Education, University of Strathclyde, Glasgow, UK. ${ }^{3}$ Department of Movement and Sports Sciences, Ghent University, Ghent, Belgium. ${ }^{4}$ Department of Sport and Exercise Sciences, Durham University, Durham City, UK. ${ }^{5}$ Department of Nutrition and Dietetics, School of Health Science and Education, Harokopio University, Athens, Greece.

Received: 26 July 2019 Accepted: 17 October 2019

Published online: 09 November 2019

\section{References}

1. Broyles S, Denstel K, Church T, Chaput J, Fogelholm M, Hu G, et al. The epidemiological transition and the global childhood obesity epidemic. Int J Obes Suppl. 2015;5(S2):S3.

2. Skinner AC, Perrin EM, Moss LA, Skelton JA. Cardiometabolic risks and severity of obesity in children and young adults. N Engl J Med. 2015;373(14): 1307-17.

3. Saldanha-Gomes C, Heude B, Charles M, de Lauzon-Guillain B, Botton J, Carles $\mathrm{S}$, et al. Prospective associations between energy balance-related behaviors at 2 years of age and subsequent adiposity: the EDEN motherchild cohort. Int J Obes. 2017;41(1):38

4. Jackson SL, Cunningham SA. The stability of children's weight status over time, and the role of television, physical activity, and diet. Prev Med. 2017; 100:229-34

5. Jouret B, Ahluwalia N, Dupuy M, Cristini C, Negre-Pages L, Grandjean H, et al. Prevention of overweight in preschool children: results of kindergarten-based interventions. Int J Obes. 2009;33(10):1075.

6. De Craemer M, De Decker E, Verloigne M, De Bourdeaudhuij I, Manios Y, Cardon G. The effect of a kindergarten-based, family-involved intervention on objectively measured physical activity in Belgian preschool boys and girls of high and low SES: the ToyBox-study. Int J Behav Nutr Phys Act. 2014; 11(1):38.

7. Reilly JJ, Kelly L, Montgomery C, Williamson A, Fisher A, McColl JH, et al. Physical activity to prevent obesity in young children: cluster randomised controlled trial. Bmj. 2006:333(7577):1041.

8. Bluford DA, Sherry B, Scanlon KS. Interventions to prevent or treat obesity in preschool children: a review of evaluated programs. Obesity. 2007;15(6): 1356-72.

9. Brown T, Moore THM, Hooper L, Gao Y, Zayegh A, ljaz S, et al. Interventions for preventing obesity in children. Cochrane Database Syst Rev. 2019;7.

10. Manios Y. The 'ToyBox-study' obesity prevention programme in early childhood: an introduction. Obes Rev. 2012;13(1):1.

11. Lambrinou $C P$, van Stralen MM, Androutsos $O$, Moreno LA, lotova $V$, Socha $P$, et al. Mediators of the effectiveness of an intervention promoting water consumption in preschool children: the ToyBox study. J Sch Health. 2018; 88(12):877-85.

12. Lambrinou C-P, van Stralen MM, Androutsos O, Cardon G, De Craemer M, lotova $V$, et al. Mediators of the effectiveness of a kindergarten-based, family-involved intervention on pre-schoolers' snacking behaviour: the ToyBox-study. Public Health Nutr. 2019;22(1):157-63.

13. Reeves S, Poh B, Essau C, Summerbell C, Cheah W, Koh D, et al. ToyBox Study Malaysia: improving healthy energy balance and obesity-related behaviours among pre-schoolers in Malaysia. Nutr Bull. 2018;43(3):290-5.

14. Malden S, Hughes AR, Gibson A-M, Bardid F, Androutsos O, De Craemer M, et al. Adapting the ToyBox obesity prevention intervention for use in Scottish preschools: protocol for a feasibility cluster randomised controlled trial. BMJ Open. 2018:8(10):e023707.

15. Craig P, Dieppe P, Macintyre S, Michie S, Nazareth I, Petticrew M. Developing and evaluating complex interventions: the new Medical Research Council guidance. BMJ. 2008;337:a1655. 
16. Eldridge SM, Chan CL, Campbell MJ, Bond CM, Hopewell S, Thabane L, et al. CONSORT 2010 statement: extension to randomised pilot and feasibility trials. BMC Pilot Feasibility Stud.2016;2(1):64.

17. Child Poverty: Glasgow Centre for Population Health; 2017. Available from: https://www.understandingglasgow.com/indicators/children/poverty/ overview. Accessed 25 Jan 2019.

18. Jackson CL, Greenhalgh T. Co-creation: a new approach to optimising research impact. Med J Aust. 2015;203(7):283-4.

19. Durlak JA, DuPre EP. Implementation matters: a review of research on the influence of implementation on program outcomes and the factors affecting implementation. Am J Community Psychol. 2008;41 (3-4):327-50.

20. Androutsos O, Apostolidou E, lotova V, Socha P, Birnbaum J, Moreno L, et al. Process evaluation design and tools used in a kindergarten-based, familyinvolved intervention to prevent obesity in early childhood. The T oy B oxstudy. Obes Rev. 2014;15:74-80.

21. Cole TJ. The LMS method for constructing normalized growth standards. Eur J Clin Nutr. 1990:44(1):45-60.

22. Cole T. Growth monitoring with the British 1990 growth reference. Arch Dis Child. 1997;76(1):47-9.

23. Group WMGRS. WHO Child Growth Standards based on length/height, weight and age. Acta Paediatr Suppl. 2006;450:76.

24. De ED, De MC, Santos-Lozano A, Van EC, Cardon G. Validity of the ActivPAL ${ }^{\text {TM }}$ and the ActiGraph monitors in preschoolers. Med Sci Sports Exerc. 2013;45(10):2002-11.

25. Addy CL, Trilk JL, Dowda M, Byun W, Pate RR. Assessing preschool children's physical activity: how many days of accelerometry measurement. Pediatr Exerc Sci. 2014;26(1):103-9.

26. De Beer M, Timmers T, Weijs PJ, Gemke RJ. Validation of total body water analysis by bioelectrical impedance analysis with deuterium dilution in (pre) school children. E-SPEN, the European e-Journal of Clinical. Nutr Metabolism. 2011;6(5):e223-e6.

27. Martin A, McNeill M, Penpraze V, Dall P, Granat M, Paton JY, et al. Objective measurement of habitual sedentary behavior in pre-school children: comparison of activPAL with Actigraph monitors. Pediatr Exerc Sci. 2011; 23(4):468-76.

28. Mouratidou T, Miguel M, Androutsos O, Manios Y, De Bourdeaudhuij I, Cardon G, et al. Tools, harmonization and standardization procedures of the impact and outcome evaluation indices obtained during a kindergartenbased, family-involved intervention to prevent obesity in early childhood: the ToyBox-study. Obes Rev. 2014:15:53-60.

29. Mouratidou T, Graffe MIM, Huybrechts I, De Decker E, De Craemer M, Androutsos $\mathrm{O}$, et al. Reproducibility and relative validity of a semiquantitative food frequency questionnaire in European preschoolers: the ToyBox study. Nutrition. 2019;65:60-7.

30. González-Gil E, Mouratidou T, Cardon G, Androutsos O, De Bourdeaudhuij I, Góźdź M, et al. Reliability of primary caregivers reports on lifestyle behaviours of E uropean pre-school children: the T oy B ox-study. Obes Rev. 2014;15:61-6.

31. Verloigne M, Ahrens W, De Henauw S, Verbestel V, Mårild S, Pigeot I, et al. Process evaluation of the IDEFICS school intervention: putting the evaluation of the effect on children's objectively measured physical activity and sedentary time in context. Obes Rev. 2015;16:89-102.

32. Pinket A-S, Van Lippevelde W, De Bourdeaudhuij I, Deforche B, Cardon G, Androutsos $\mathrm{O}$, et al. Effect and process evaluation of a cluster randomized control trial on water intake and beverage consumption in preschoolers from six European countries: the ToyBox-study. PLoS One. 2016;11(4): e0152928.

33. Abbott JH. The distinction between randomized clinical trials (RCTs) and preliminary feasibility and pilot studies: what they are and are not. JOSPT, Inc. JOSPT, 1033 North Fairfax Street, Suite 304, Alexandria, VA ... ; 2014.

34. Moore GF, Audrey S, Barker M, Bond L, Bonell C, Hardeman W, et al. Process evaluation of complex interventions: Medical Research Council guidance. BMJ. 2015;350:h1258.

35. Langford R, Jago R, White J, Moore L, Papadaki A, Hollingworth W, Metcalfe C, Ward D, Campbell R, Wells S, Kipping R. A physical activity, nutrition and oral health intervention in nursery settings: process evaluation of the NAP SACC UK feasibility cluster RCT. BMC PublicHealth. 2019;19(1):865.

36. Latomme J, Cardon G, De Bourdeaudhuij I, lotova V, Koletzko B, Socha P, et al. Effect and process evaluation of a kindergarten-based, family-involved intervention with a randomized cluster design on sedentary behaviour in 4- to 6-year old European preschool children: the ToyBox-study. PLoS One. 2017;12(4):e0172730

37. Barber SE, Jackson C, Hewitt C, Ainsworth HR, Buckley H, Akhtar S, et al. Assessing the feasibility of evaluating and delivering a physical activity intervention for pre-school children: a pilot randomised controlled trial. Pilot Feasibility Stud. 2016;2(1):12.

38. Ginja S, Arnott B, Araujo-Soares V, Namdeo A, McColl E. Feasibility of an incentive scheme to promote active travel to school: a pilot cluster randomised trial. Pilot Feasibility Stud. 2017;3(1):57.

39. Lloyd JJ, Wyatt KM, Creanor S. Behavioural and weight status outcomes from an exploratory trial of the Healthy Lifestyles Programme (HeLP): a novel school-based obesity prevention programme. BMJ Open. 2012;2(3): e000390.

40. Brown B, Harris KJ, Heil D, Tryon M, Cooksley A, Semmens E, et al. Feasibility and outcomes of an out-of-school and home-based obesity prevention pilot study for rural children on an American Indian reservation. Pilot Feasibility Stud. 2018;4(1):129.

41. Knowlden A, Sharma M. Systematic review of family and home-based interventions targeting paediatric overweight and obesity. Obes Rev. 2012; 13(6):499-508.

42. Heinrichs N, Bertram H, Kuschel A, Hahlweg K. Parent recruitment and retention in a universal prevention program for child behavior and emotional problems: barriers to research and program participation. Prev Sci. 2005;6(4):275-86.

43. Plueck J, Freund-Braier I, Hautmann C, Beckers G, Wieczorrek E, Doepfner M. Recruitment in an indicated prevention program for externalizing behaviorparental participation decisions. Child Adolesc Psychiatry Ment Health. 2010; 4(1):15.

44. McDonald L, FitzRoy S, Fuchs I, Fooken I, Klasen H. Strategies for high retention rates of low-income families in FAST (families and schools together): an evidence-based parenting programme in the USA, UK, Holland and Germany. Eur J Dev Psychol. 2012;9(1):75-88.

45. Sacher PM, Kolotourou M, Chadwick PM, Cole TJ, Lawson MS, Lucas A, et al. Randomized controlled trial of the MEND program: a family-based community intervention for childhood obesity. Obesity. 2010;18(S1):S62-S8.

46. Hughes AR, Stewart L, Chapple J, McColl JH, Donaldson M, Kelnar C, et al. Randomized, controlled trial of a best-practice individualized behavioral program for treatment of childhood overweight: Scottish childhood overweight treatment trial (SCOTT). Pediatrics. 2008;121(3):e539-e46.

47. Talma H, Chinapaw M, Bakker B, HiraSing R, Terwee C, Altenburg T. Bioelectrical impedance analysis to estimate body composition in children and adolescents: a systematic review and evidence appraisal of validity, responsiveness, reliability and measurement error. Obes Rev. 2013;14(11): 895-905.

48. Kakinami L, Henderson M, Chiolero A, Cole TJ, Paradis G. Identifying the best body mass index metric to assess adiposity change in children. Arch Dis Child. 2014;99(11):1020-4.

49. Reilly J, Dorosty A, Emmett P. Identification of the obese child: adequacy of the body mass index for clinical practice and epidemiology. Int J Obes. 2000;24(12):1623.

50. Howie EK, Straker LM. Rates of attrition, non-compliance and missingness in randomized controlled trials of child physical activity interventions using accelerometers: a brief methodological review. J Sci Med Sport. 2016:19(10): 830-6.

51. Jones RA, Riethmuller A, Hesketh K, Trezise J, Batterham M, Okely AD. Promoting fundamental movement skill development and physical activity in early childhood settings: a cluster randomized controlled trial. Pediatr Exerc Sci. 2011;23(4):600-15.

52. Stark LJ, Spear S, Boles R, Kuhl E, Ratcliff M, Scharf C, et al. A pilot randomized controlled trial of a clinic and home-based behavioral intervention to decrease obesity in preschoolers. Obesity. 2011;19(1):134-41.

53. Shi Y, Huang WY, Yu JJ, Sheridan S, Sit CH-P, Wong SH-S. Compliance and practical utility of continuous wearing of activPAL ${ }^{\text {TM }}$ in adolescents. Pediatr Exerc Sci. 2019:00:1-7.

54. De Craemer M, Lateva M, lotova V, De Decker E, Verloigne M, De Bourdeaudhuij I, et al. Differences in energy balance-related behaviours in European preschool children: the ToyBox-study. PLoS One. 2015;10(3): e0118303.

55. Buzzard IM, Stanton CA, Figueiredo M, Fries EA, Nicholson R, Hogan CJ, et al. Development and reproducibility of a brief food frequency 
questionnaire for assessing the fat, fiber, and fruit and vegetable intakes of rural adolescents. J Am Diet Assoc. 2001;101(12):1438-46.

56. Koleilat M, Whaley SE. Reliability and validity of food frequency questions to assess beverage and food group intakes among low-income 2-to 4-year-old children. J Acad Nutr Diet. 2016;116(6):931-9.

57. Dani K, Stobo D, Capell H, Madhok R. Audit of literacy of medical patients in North Glasgow. Scott Med J. 2007;52(2):21-4.

58. Macintyre S, Macdonald L, Ellaway A. Do poorer people have poorer access to local resources and facilities? The distribution of local resources by area deprivation in Glasgow, Scotland. Soc Sci Med. 2008;67(6):900-14.

59. Knai C, Lobstein T, Darmon N, Rutter H, McKee M. Socioeconomic patterning of childhood overweight status in Europe. Int J Environ Res Public Health. 2012;9(4):1472-89.

\section{Publisher's Note}

Springer Nature remains neutral with regard to jurisdictional claims in published maps and institutional affiliations.

Ready to submit your research? Choose BMC and benefit from:

- fast, convenient online submission

- thorough peer review by experienced researchers in your field

- rapid publication on acceptance

- support for research data, including large and complex data types

- gold Open Access which fosters wider collaboration and increased citations

- maximum visibility for your research: over $100 \mathrm{M}$ website views per year

At BMC, research is always in progress.

Learn more biomedcentral.com/submissions 